\title{
Norois
}

Environnement, aménagement, société

$208 \mid 2008 / 3$

Gestion du littoral, risques urbains, ours en Pyrénées

\section{Le tourisme macabre à La Nouvelle-Orléans après Katrina : résilience et mémorialisation des espaces affectés par des catastrophes majeures}

Dark tourism in post Katrina New Orleans: resilience and memory at major disaster sites

Julie Hernandez

\section{(2) OpenEdition}

Journals

Édition électronique

URL : https://journals.openedition.org/norois/2208

DOI : $10.4000 /$ norois. 2208

ISBN : 978-2-7535-1556-7

ISSN : 1760-8546

Éditeur

Presses universitaires de Rennes

Édition imprimée

Date de publication : 1 novembre 2008

Pagination : 61-73

ISBN : 978-2-7535-0788-3

ISSN : 0029-182X

Référence électronique

Julie Hernandez, « Le tourisme macabre à La Nouvelle-Orléans après Katrina : résilience et mémorialisation des espaces affectés

par des catastrophes majeures », Norois [En ligne], 208 | 2008/3, mis en ligne le 01 novembre 2010 consulté le 14 janvier 2022. URL : http://journals.openedition.org/norois/2208 ; DOI : https://doi.org/ $10.4000 /$ norois. 2208 


\title{
Le TOURISME MACABRE À LA NOUVELle-OrLÉANS APRÈS KATRINA : RÉSILIENCE ET MÉMORIALISATION DES ESPACES AFFECTÉS PAR DES CATASTROPHES MAJEURES
}

\author{
JuLie HERNANDEZ \\ Doctorante en Géographie - GECKO EA 375 \\ (Université de Paris X Nanterre), \\ 200 avenue de la République - 92001 NANTERRE Cedex \\ hernanjulie@gmail.com
}

\begin{abstract}
RÉSUMÉ
Le "tourisme macabre » renvoie à des pratiques de tourisme culturel, centrées sur la visite des sites de mort et de dévastation. Après avoir exposé l'histoire de ce concept et les multiples réalités qu'il recouvre, cette recherche s'attache à examiner comment cette forme de tourisme s'est rapidement développée à La Nouvelle-Orléans après le passage de l'ouragan Katrina, le 29 août 2005. Il s'agit ici d'analyser les enjeux multiples et parfois conflictuels de ces pratiques, à travers l'étude des motivations et des interactions entre les différents acteurs de ce tourisme macabre.

Ce dernier participe en effet de la patrimonialisation des espaces affectés par la catastrophe, mais ce faisant, il met également en lumière les enjeux politiques, sociaux et culturels, liés à l'interprétation de l'événement et aux discours sur la reconstruction de la ville.

Les nécessités économiques et l'horizon d'attente des touristes par rapport aux représentations de La Nouvelle-Orléans dans la conscience collective américaine conduisent ainsi à la construction d'une mémoire sélective de la catastrophe. Cela se traduit par une surreprésentation de certains lieux et, corrélativement, par un manque de visibilité d'autres espaces dont les besoins peinent à accéder à la sphère publique.
\end{abstract}

Mots CLÉ : tourisme macabre - La Nouvelle-Orléans - catastrophe naturelle-ouragan Katrina - processus de mémorialisation

\footnotetext{
ABSTRACT

Dark tourism in post Katrina New Orleans: resilience and memory at major disaster sites

"Dark tourism" is a form of cultural tourism that focuses on the visit of death and devastation sites. After describing the scientific background of this concept and the many situations it covers, this research will try to study how dark tourism practices quickly developed in post-Katrina New Orleans. The point here is to analyze the multiple, and sometime conflicting, issues at stake through these practices. We will do so by observing the motivations and relations between the participants, producers, and witnesses of these "Katrina tours".
} 
These tours are part of a cultural capitalization on the disaster, but also highlight the political, social, and cultural issues regarding its interpretation and the discourses on the city recovery.

Economic necessities as well as the tourists'expectations vis-à-vis certain representations of New Orleans in the American psyche led to the creation of a selective memory of the disaster. This translates into an overrepresentation of some neighborhoods and, conversely, into a lack of visibility of other places whose needs do not appear in the public sphere.

KEY WORDS : dark tourism - New Orleans - natural disaster - hurricane Katrina - disaster memory

Le passage de l'ouragan Katrina le 29 août 2005 et ses conséquences à La Nouvelle-Orléans ont, à bien des égards, fait l'effet d'un « 11 septembre intérieur » aux États-Unis. Le nombre de morts, officiellement arrêté à 1300 , et l'étendue des destructions, $80 \%$ de la ville noyée sous les eaux du Pontchartrain, suffiraient à classer Katrina dans la catégorie des catastrophes hors normes. Mais la comparaison avec ce qui s'est passé en 2001 vaut aussi et surtout par la similarité des traumatismes causés par ces deux événements sur les représentations que les Américains se faisaient du monde extérieur après New York, (cf. Stein, 2003), de leur propre territoire après La Nouvelle-Orléans. Troisième grande catastrophe médiatique du XXI ${ }^{\mathrm{e}}$ siècle, après le 11 septembre et le tsunami en Asie du Sud-Est, les scènes de la première semaine de septembre 2005 ont révélé, bien plus que généré, l'existence d'un «tiers-monde » au sein même de la première puissance mondiale : pauvreté extrême de populations restées prisonnières d'espaces urbains oubliés, faillite des systèmes de protection technique, faillite surtout des pouvoirs publics, incapables de venir secourir leurs propres citoyens. Les victimes réfugiées sur leurs toits émergeant à peine de l'eau, les maisons balayées trois rues plus loin et les drapeaux américains agités à l'envers en signe de détresse ont suffisamment marqué la psyché américaine pour que beaucoup décident de venir à La Nouvelle-Orléans pour voir de leurs yeux les destructions laissées par l'ouragan.

La visite guidée des quartiers détruits et Katrina Tours ont été organisés dès octobre 2007 par des acteurs de l'industrie touristique déjà présents dans la ville avant le passage de l'ouragan. Exploitation commerciale d'une curiosité malsaine ou nécessaire pèlerinage destiné à marquer les esprits du grand public, toujours prompt à déplacer son attention vers la catastrophe suivante, cette pratique constitue aussi un formidable vecteur d'information sur l'état de la ville après Katrina, via les quartiers visités, photographiés et plus tard peut-être racontés. Les enjeux s'organisant autour de ces espaces supports sont multiples et parfois conflictuels, par rapport aux contenus symboliques attribués aux lieux visités, mais aussi aux significations de cette pratique elle-même. Catharsis et objectivisation du désastre, devenu simple curiosité touristique, impératifs économiques et reconstruction d'une image positive de la ville, construction et instrumentalisation d'une mémoire de la catastrophe sont autant de fonctions attribuées à des lieux symboles en devenir. Ces lieux sont produits, investis, consommés par les clients et les marchands de l'industrie touristique mais aussi par les acteurs locaux, dont les conceptions et les usages des espaces urbains s'opposent parfois diamétralement. Pourtant les lignes qui séparent les combattants et les enjeux de ces " guerres culturelles » (Mitchell, 2000) pour l'appropriation des espaces de la catastrophes ne se résument pas nécessairement à une opposition entre touristes « consommateurs - voyeurs » et victimes révoltées par la commercialisation du spectacle de leurs souffrances.

Les tensions autour de la patrimonialisation de l'événement agitent également les acteurs, individuels et collectifs, de la reconstruction. Le possible consensus autour d'un statut unificateur, socialement et spatialement, de victimes d'une hypothétique et irascible " Mère Nature », n'a pas tenu devant la certitude très tôt installée que « Katrina » n'avait rien d'une catastrophe « natu- 
relle ${ }^{1} »$. En ce sens, l'insistance sur la violence et l'unicité de l'aléa cyclonique, marqués dans certains discours sur l'interprétation des évènements de l'été 2005, ne produit pas une mémoire neutre de la catastrophe, mais une mémoire neutralisante vis-à-vis des enjeux sociaux qui fondaient la vulnérabilité de La Nouvelle-Orléans. De sorte que la patrimonialisation du désastre, étudiée ici à travers le prisme de la mise en tourisme des quartiers dévastés (fig. 1), doit être considéré moins comme une patrimonialisation de l'événement naturel, que comme un exemple de mise en scène des tensions sociales et culturelles similaires à celles qui organisent la production d'une mémoire controversée d'évènements historiques et politiques majeurs.

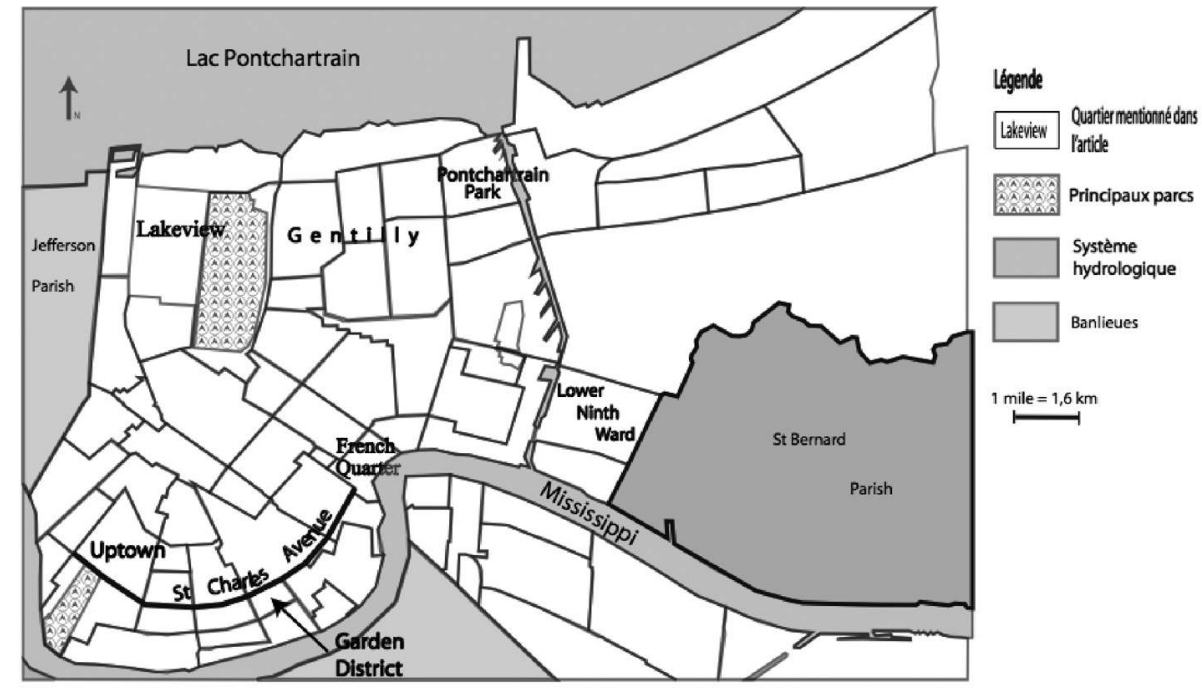

Figure 1 : Localisation des principaux quartiers cités dans l'article et niveau d'inondation au $1^{\text {er }}$ septembre 2005 (Source : Greater New Orleans Center for Data Collection. Cartographie par l'auteur)

September $1^{\text {st }}, 2005$

Situation of the neighborhoods mentioned in the article and levels of flooding on

Les données utilisées dans cet article sont tirées d'un long travail d'observation participante sur le terrain (août 2005-juillet 2007). Une revue de presse a été réalisée dans les journaux locaux et nationaux pour mesurer et analyser l'écho de ces pratiques de tourisme macabre dans les médias, et donc dans le grand public. Des enquêtes systématiques ont été menées en février-mars 2007, portant sur les motivations, les perceptions et les réactions de 47 participants aux Katrina Tours (24 sur le circuit proposé par I. Cossart, 23 sur celui offert par Gray Line), en essayant, autant que possible, de respecter des ratios hommes/femmes, et Blancs/Noirs/Hispaniques proches des moyennes nationales américaines. En contrepoint, 50 habitants de La Nouvelle-Orléans ont été interrogés sur leurs réactions devant cette mise en tourisme de la catastrophe. Les proportions hommes/femmes, Blancs/Noirs de la ville, post-Katrina, ont été conservés et les personnes interrogées ont été sélectionnées dans une dizaine de quartiers différemment affectés par la catastrophe.

1. M. Kofi Annan déclarait aux New York Times dès le 4 septembre 2005 que Katrina devait être considérée comme une "man-made catastrophe". 


\section{Tourisme macabre, disaster marketing : des pratiques loin d'être inédites}

\section{LE TOURISME MACABRE ENTRE VOYEURISME ET CATHARSIS}

« Il faut le voir pour le croire. » Les valeurs éducatives et cathartiques de l'expérience directe de la scène d'un miracle ou d'un drame sont reconnues depuis les premiers pèlerinages religieux jusqu'aux touristes silencieusement massés devant les grillages de Ground Zero (Yuill, 2004). Cette pratique est loin d'être inédite, aux États-Unis comme dans le reste du monde : les champs de bataille de la Guerre de Sécession américaine étaient visités par des « groupes de mémoire » dès la fin du XIX ${ }^{e}$ siècle (Weeks, 2003), de même qu'Hiroshima et Nagasaki figurent en bonne place dans les destinations touristiques du Japon (Yuill, 2004). La visite des sites associés à la mort, aux catastrophes, aux tragédies et aux atrocités humaines, est en fait une forme particulière de tourisme culturel, ce dernier étant défini comme un voyage dont le but est de faire l'expérience directe des arts, des héritages et du caractère particulier, d'autres diraient de « l'esprit », d'un lieu. Derrière ce concept de « tourisme macabre » (dark tourism, Foley et Lennon, 2003) ou thanatourisme (Seaton, 1996) sont rassemblés des lieux et des pratiques très diverses, puisqu'il désigne aussi bien les promenades dans les cimetières historiques de certaines villes (le Père Lachaise à Paris, St Louis Cemetery $n^{\circ} 1$ à La Nouvelle-Orléans) que les pèlerinages des survivants de la Shoah vers les camps d'extermination d'Europe de l'Est. Lennon et Foley (2003) proposent de réduire cette définition aux pratiques touristiques pour lesquelles la visite des sites de mort ou de dévastation constituent le but premier du voyage. Cette restriction permet de mettre en évidence le développement touristique de villes dont la vocation dans ce domaine était improbable avant qu'une catastrophe n'y survienne. Oklahoma City, est un bon exemple des conséquences économiques, sociales mais aussi culturelles de cette soudaine acquisition d'une identité urbaine tragique. Quasi inconnue sur la carte des destinations touristiques américaines jusqu'au attentats de mai 1995, la ville tire, depuis la création du mémorial, près de $12 \%$ de ses revenus annuels du tourisme, et s'est hissée à la $9^{e}$ place du classement des villes les plus « significatives » pour les Américains ${ }^{2}$ (Linenthal, 2005).

La définition de Foley et Lennon pose en revanche problème quand les sites affectés par un désastre étaient déjà d'importantes destinations touristiques, comme cela a pu être le cas à New York et plus significativement encore à La Nouvelle-Orléans. Il est en effet difficile d'isoler dans ces villes ce qui relève de pratiques touristiques classiques investissant un espace inédit (un quartier détruit par exemple), et ce qui est strictement de l'ordre du « tourisme macabre ». La question des motivations des acteurs est bien entendue essentielle pour développer cette distinction. Bien que ces formes de tourisme soient souvent dénoncées comme le produit d'une curiosité morbide, les raisons de leur visite sur les lieux dévastés par Katrina allaient, pour une cinquantaine de touristes interrogés durant l'hiver 2006 - 2007, de leur intérêt pour l'histoire et la culture à la perception d'un devoir de témoignage et de mémoire.

\section{DE LA NÉCESSITÉ D’UN « RETOUR À LA NORMALE » : \\ RÉSILIENCE ÉCONOMIQUE, RÉSILIENCE DES REPRÉSENTATIONS URBAINES}

À La Nouvelle-Orléans, cette exploitation de la catastrophe des fins touristiques s'est très rapidement imposée dans la continuité des pratiques de marketing urbain propres à la ville depuis le début du XX siècle (Stanonis, 2006). T-shirts commémoratifs ${ }^{3}$ et autocollants ironisant sur les pillages ou l'ineptie des autorités ${ }^{4}$ sont une des expressions de ce Katrina business. Les disaster

2. Chaque année un sondage s’intéresse aux villes que les citoyens américains considèrent comme "the most meaningful for the country".

3. "I survived Hurricane Katrina".

4. "I stayed in New Orleans during Katrina and all I got is this t-shirt, a new cadillac and a plasma TV", "FEMA : Federal Employees Missing Again". 
tours, c'est-à-dire les visites guidées des quartiers affectés par la catastrophe, sont apparus dès octobre 2005. Destinés à l'origine aux employés fédéraux et aux ingénieurs chargés de la reconstruction, ils se sont ensuite étendus aux familles de ces mêmes employés et ingénieurs, avant de faire officiellement partie des packages offerts aux touristes à la fin de l'automne 2005. Ces tours reposent sur des initiatives privées, menées par des acteurs déjà impliqués dans l'économie touristique de la ville. La première à s'être lancée fut Isabelle Cossart, une française émigrée à La Nouvelle-Orléans qu'elle fait découvrir aux touristes depuis plus de trente ans. Son minibus promenant les touristes dans les quartiers détruits a vite été rejoint par les cars des tours opérateurs régionaux et nationaux comme Gray Line. Le Bureau du Tourisme de la ville a confirmé, en janvier 2007, le succès de ces visites guidées et l’importance des revenus qu'elles génèrent. Pour Isabelle Cossart, c'est la survie de son entreprise qui s'est jouée, avec succès, à l'automne 2005.

La première, et la plus évidente, fonction de cette mise en tourisme des espaces affectés par la catastrophe est la résilience économique de la ville (figure 2). Le tourisme macabre repose en effet sur une capitalisation de la valeur symbolique des lieux concernés et des flux qu'elle draine. Or le tourisme constitue, depuis les années 1980, le moteur de La Nouvelle-Orléans (Lewis, 2003. Hernandez, 2006). Ce pilier économique et social repose sur la particularité des cultures et des paysages urbains de la ville par rapport au reste du pays : architecture urbaine particulière héritée d'une très vieille colonisation européenne, usage "caribéens » de la rue destinée à bien d'autres fonctions que la simple circulation, mélanges des musiques, des cuisines et des célébrations publiques qui donnent l'impression (l'illusion?) d'une mixité sociale, raciale et culturelle sans équivalent aux États-Unis. Bien avant le passage de l'ouragan, l'espace urbain néo-orléanais était à vendre comme spectacle, paysages et pratiques.

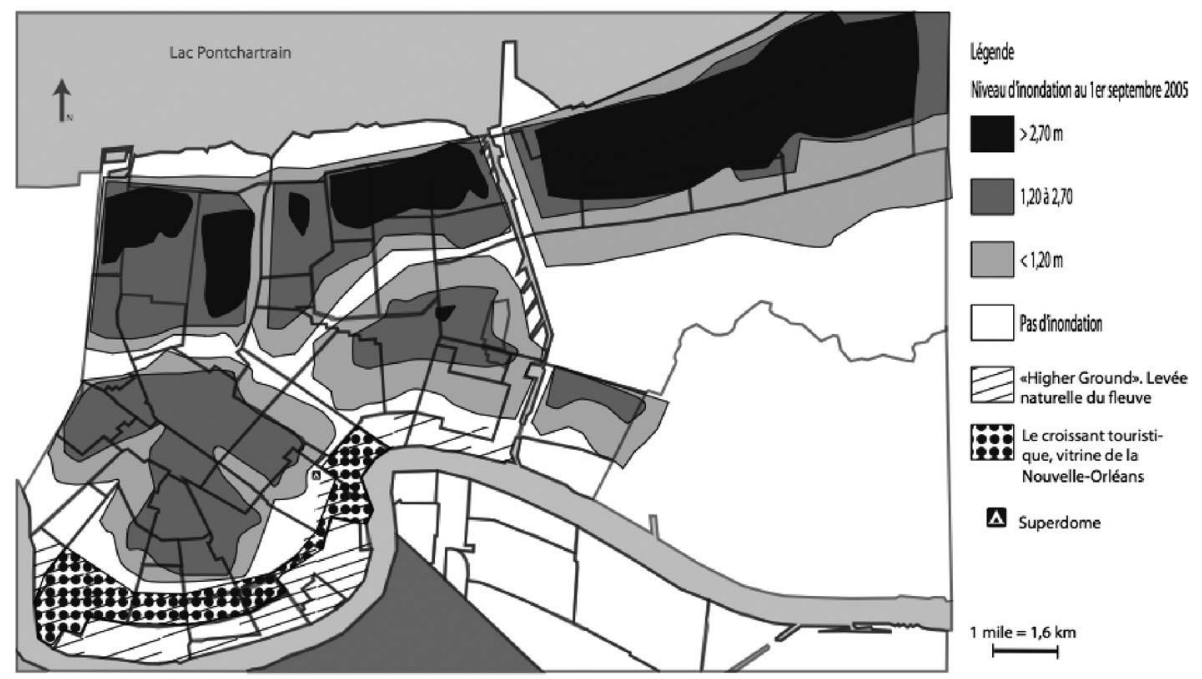

Figure 2 : La «vitrine » et «l'arrière-boutique » : une mise en tourisme sélective de la ville (Cartographie par l'auteur)

The window and the back shop : a selective development of tourism in the city

De ce fait, les acteurs, les structures et les modalités de la mise en tourisme des quartiers détruits étaient déjà en place en septembre 2005. "Katrina » n'était qu'un thème supplémentaire à ajouter aux «menus » des visites guidées thématiques, à côté des "cemeteries tours ", «Plantations tours" et autres "Vodoo tours". Les billets s'achètent dans les mêmes agences du French Quarter, les circuits ont simplement été allongés au-delà des rues historiques du «Vieux Carré » et de Bayou St John pour inclure le tristement célèbre Lower Ninth Ward, et 
plus rarement, Lakeview ou Gentilly (Fig. 1). La visite de la ville proposée par I. Cossard durait environ une heure trente en 2005, elle prend aujourd'hui trois heures. Sa brochure publicitaire était, il y a deux ans, illustrée par la photographie d'une jeune femme en costume traditionnel du Sud posant devant Oak Alley Plantation (la visite des plantations et des bayous louisianais étant toujours incluse dans les packages proposés aux touristes de passage à La Nouvelle-Orléans). Aujourd'hui cette même illustration est deux fois plus petite et deux autres photographies y ont été ajoutées : une image aérienne de l'ouragan et une photo d'une maison du Lower Ninth Ward éventrée et effondrée après le passage de Katrina. Les lieux symboles de Katrina sont ainsi intégrés aux circuits touristiques et vendus selon les mêmes modalités que les autres produits culturels dont dépend l'économie de la ville.

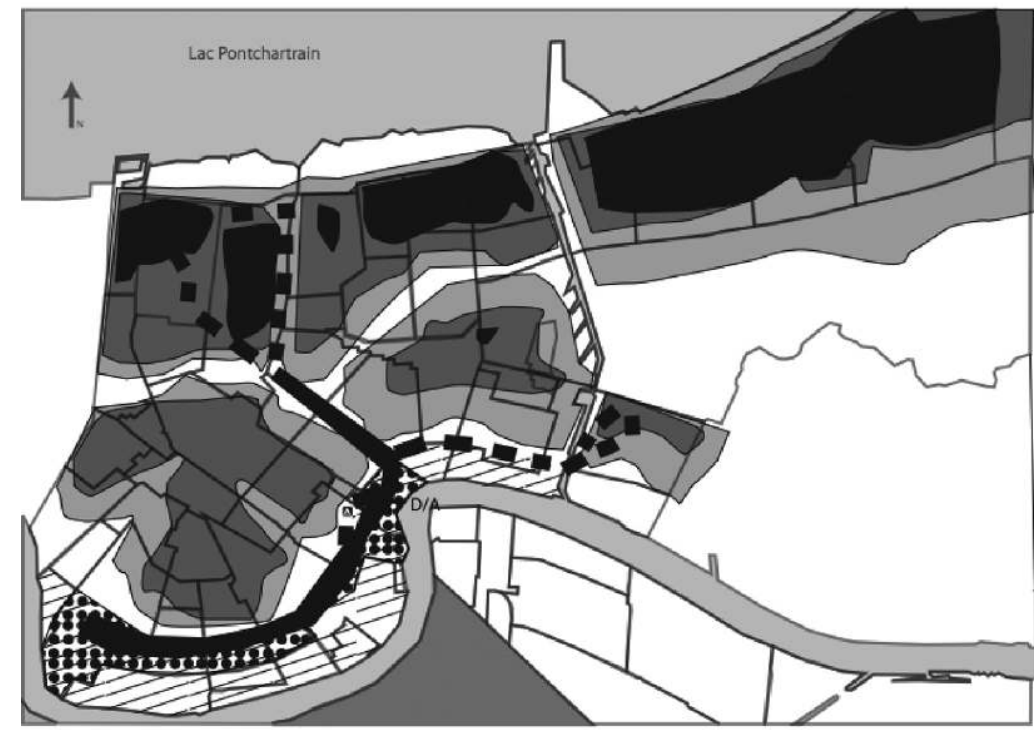

(Cartographie par l'auteur) An extension of the usual guided tour : the road of I. Cossart's tours in 2004 and 2006

Cette continuité avec les pratiques antérieures, fait écho à la perception d'une majorité des participants à ce "tourisme macabre » qui s'écarte de la définition donnée plus haut en ce que la visite de ces quartiers détruits est rarement le but premier ou unique de la venue des touristes concernés à la Nouvelle-Orléans. D’après une enquête réalisée durant l'hiver 2007 auprès d'une cinquantaine de participants aux tours organisés par I. Cossart et par Gray Line, la visite des lieux de la catastrophe n'était qu'une activité prévue parmi d'autres au cours de leur séjour en ville. Cette impression d'une consommation passive de l'espace urbain détruit, devenu une attraction comme une autre, doit cependant être nuancée par le besoin systématiquement exprimé d'être les témoins directs de ce qu'ils n'avaient jusque là vu qu'à la télévision. De plus, quelles qu'en soient les modalités, les séjours à La Nouvelle-Orléans sont le plus souvent présentés comme un devoir citoyen de solidarité, une volonté de participer à la reconstruction. Que ce soit sur Bourbon St ou dans les bus sillonnant le Lower Ninth Ward nombreux sont les touristes à reprendre les propos de cette famille venue de Californie en février 2007 : « Nous devions partir au Costa Rica cet hiver mais après ce que nous avons vu à la télévision, nous nous sommes dit qu'il vaudrait mieux 
dépenser notre argent ici. La ville et les gens d'ici en ont besoin et nous, nous passons quand même des vacances très "exotiques". »

La relance de l'activité touristique est une nécessité cruciale pour l'ensemble de la ville. Les inondation qui ont suivit le passage de Katrina ont en effet «noyé » son moteur économique. L'impact global de la catastrophe sur l'industrie touristique est difficilement quantifiable mais certains indicateurs sont dramatiques : les pertes nettes pour le seul mois de septembre 2005 avoisinent les 800 millions de dollars. Plusieurs grands évènements n'ont pu avoir lieu dont le $3^{\mathrm{e}}$ festival le plus fréquenté par les touristes, Southern Decadence, mais aussi le Essence Blues Festival, ainsi que les évènements sportifs et musicaux hébergés presque chaque semaine au Superdome. Les artistes locaux tout comme une bonne partie des employés de l'industrie touristique ont été dispersés à travers tout le pays et il a été estimé que 70 à $80 \%$ des institutions culturelles touchées par la catastrophe n'avaient toujours pas rouvert leurs portes un an après (BNOB, 2006). Face à cette situation plus qu'alarmante, les premières protestations devant l'exploitation "macabre ", «voyeuriste », des lieux détruits se sont assez vite tues devant l'idée, qu' « il fallait bien vendre quelque chose ». D'autant qu'au-delà des retombées économiques immédiates de cette reprise du tourisme, c'est toute l'image de la ville qui bénéficie de cette impression de «business as usual ». L'économie touristique est par nature extrêmement sensible à toute une géographie des représentations qui définit les lieux attractifs ou répulsifs. Les dégâts occasionnés de ce point de vue par la surmédiatisation du délabrement physique mais aussi social de la ville immédiatement après le passage de l'ouragan sont énormes. Cependant une partie de l'attractivité de La Nouvelle-Orléans repose, dans l'imaginaire collectif américain, sur le caractère permissif mais aussi désinvolte et fataliste de la ville. En ce sens, le tourisme macabre participe d'un processus de résilience par auto-conviction en servant de support à des pratiques et des discours affirmant un certain « retour à la normale ».

\section{Construction et appropriation des lieux de mémoire de la catastrophe}

\section{«SOUVENIRS » TOURISTIQUES ET MÉMORIALISATION LOCALE SPONTANÉE}

Au-delà de ces effets d'entraînement économique, ces Katrina tours jouent aussi un rôle important dans les processus de mémorialisation et de réappropriation des espaces urbains après la catastrophe. Plus de deux ans après le passage de l'ouragan, il n'existe toujours pas, à La Nouvelle-Orléans, de mémorial public officiel de la catastrophe. Plusieurs projets ont été proposés puis abandonnés, en raison de désaccords concernant les lectures du désastre, les acteurs et surtout les lieux à mettre en avant. Les nécessités économiques, nous venons de le voir, comme l'imbroglio des responsabilités politiques, tirent le souvenir de la catastrophe vers l'oubli ou du moins une indifférente exploitation commerciale. La sélectivité des dommages causés par l'ouragan et de leurs conséquences à long terme empêche tout consensus entre «victimes » revenues en ville et confrontées au quotidien de l'après Katrina et les quelques 200000 personnes toujours « déplacées » dans le reste des États-Unis. Dans quel lieu enfin exprimer la mémoire collective du désastre? La Nouvelle-Orléans dans son ensemble souffre de ce défaut de centralité typique des villes américaines, le vieux carré historique du French Quarter n'étant que l'illusion d'un centre ville largement abandonné aux touristes, et le CBD une concentration de bureaux appartenant aux firmes « allogènes » venues du Texas ou d'ailleurs. Au-delà de ces deux espaces mitoyens s'étend une « ville de quartiers » où il n'est pas rare de trouver plusieurs générations d'une même famille sur quelques rues, où l'appartenance locale est revendiquée, pratiquée à travers les fêtes de rue et la fréquentation de l'église ou des restaurants et bars locaux. C'est à cette échelle que la mémoire de la catastrophe de l'été 2005 se donne à voir. Ici les autocollants financés par les associations locales proclament la survie de Broadmoor ${ }^{5}$, l'un des quartiers les plus durement touché par les 
inondations. Parfois ce sont les croix peintes sur les portes des maisons par les organisations de secours (signalant la date de leur passage et le nombre de cadavres découverts à l'intérieur) qui n'ont, d'un commun accord, pas été recouvertes. Ailleurs encore, là où le passage de l'ouragan a tout balayé, ce sont de petits objets commémoratifs, photos, trophées sportifs qui sont posés sur les perrons en béton, parfois les seuls éléments ayant survécu à la catastrophe (photo 1). Or la portée de ces mémoires spontanées, fragmentées, de la catastrophe dépend aussi de leur visibilité, de leur réception et de leur « enregistrement » par le reste de la population. De ce point de vue, les visiteurs de passage à la Nouvelle-Orléans jouent un rôle de témoins, et permettent d'articuler les traces laissées par Katrina ou par les habitants en souvenir de la catastrophe, sur l'espace urbain à la conscience collective américaine (Lipztig, 1990. Eyre et al., 2000).

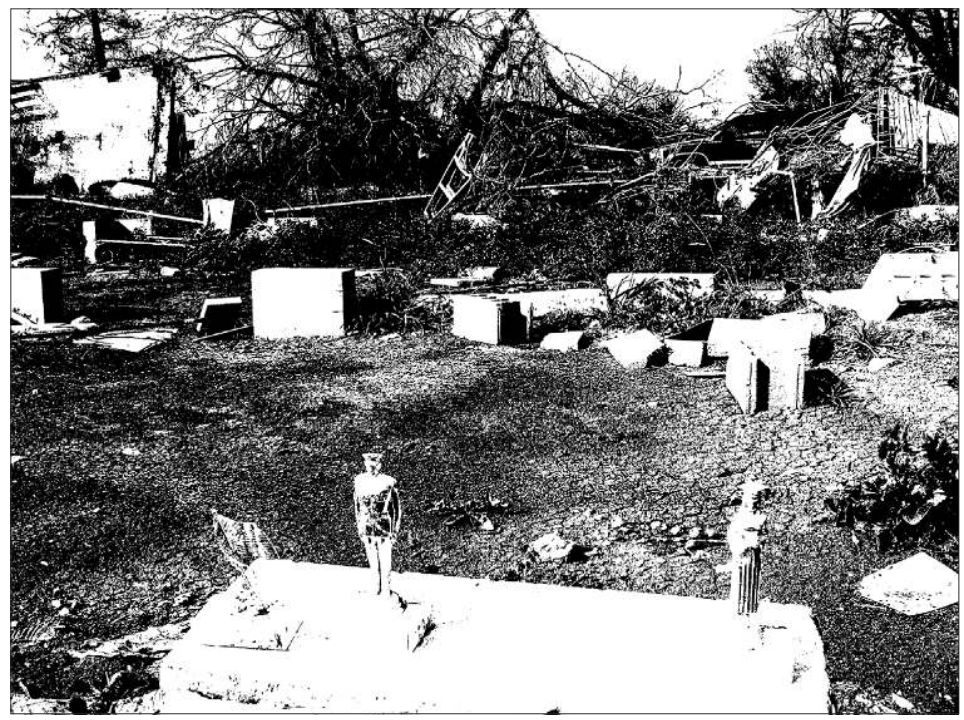

Photo 1 : Une mémorialisation spontanée de la catastrophe (Lower Ninth Ward, décembre 2005 - cliché de l'auteur)

Creating a spontaneous memory of the disaster

Quelles sont donc les capacités d'une pratique spatiale, la visite guidée, à vocation économique d'une part et de loisir d'autre part, à rendre compte des réalités urbaines de l'après-Katrina à La Nouvelle-Orléans? Les circuits touristiques proposés sont en effet soumis à certains impératifs. Dans la mesure où ils ne sont que rarement le but premier du voyage, souvent de courte durée ${ }^{6}$, ils doivent être limités dans le temps pour laisser la place à d'autres activités touristiques. Ceci entraîne nécessairement une limitation du nombre de lieux visités. De plus, le format des visites (trajets en car avec quelques pauses photographiques) ne favorisent pas les interactions avec les populations locales éventuellement revenues dans les quartiers. Ces dernières ne sont d'ailleurs jamais impliquées dans l'élaboration de ces tours, et ne bénéficient pas directement des revenus de ces activités.

Mais surtout, ce tourisme de catastrophe se doit de répondre à un horizon d'attente des visiteurs venus constater les dégâts laissés par Katrina, de sorte que deux types de lieux sont privilégiés par ces tours pour répondre à cette exigence : ceux qui sont immédiatement identifiables par les touristes et ceux où le niveau de destruction est suffisamment spectaculaire pour qu'ils deviennent des curiosités touristiques.

6. D’après le Bureau du Tourisme de la ville, la durée moyenne des séjours touristiques à la Nouvelle-Orléans est de 4 jours. 


\section{QUARTIERS « MARTYRS » ET LIEUX OUBLIÉS :}

\section{DU TOURISME MACABRE COMME PRATIQUE CRÉATRICE DE SYMBOLES}

Cette double contrainte conduit à une surreprésentation de certains espaces. Parmi ceux-ci on trouve les « hauts lieux » de la catastrophe, tels qu'ils ont été construits par la représentation médiatique. Le Convention Center et surtout le Louisiana Superdome, en sont les deux plus flagrants exemples. Tous deux sont situés à proximité du French Quarter, donc de l'épicentre touristique de La Nouvelle-Orléans. Leur visite constitue par conséquent une extension facile des tours proposés au départ du «Vieux Carré ». De plus, dans le cas du Superdome en particulier, l'architecture immédiatement reconnaissable du stade a favorisé le développement de sa dimension symbolique dans l'imaginaire américain. Il est intéressant de noter que les visiteurs de la NouvelleOrléans associaient toujours en 2006 ce bâtiment au souvenir de Katrina, tandis que pour les populations locales, y compris pour certains anciens « réfugiés » du stade au moment de l'ouragan, le Superdome renvoyait plutôt à des images positives, comme lieu de sociabilité et de solidarité de la ville autour de son équipe de football ${ }^{7}$. Certains hauts-lieux médiatiques de la catastrophe sont donc ignorés par les processus de mémorialisation locaux. Si la Second Line ${ }^{8}$ spontanée du premier anniversaire de la catastrophe (2006) avait emprunté le trajet menant du Superdome au Convention Center, toutes les marches commémoratives d'août 2007, partaient de, et traversaient des quartiers encore majoritairement détruits deux ans après le passage de l'ouragan.

Le statut de ces quartiers est beaucoup plus problématique dans leur réinvestissement par les acteurs du «tourisme macabre ». Ce dernier tend en effet à se concentrer sur les espaces où les destructions sont les plus spectaculaires, c'est-à-dire le plus souvent sur les quartiers situés à proximité des points de rupture des levées, là où la force de l'eau a balayé et mis sans dessusdessous constructions, voitures et végétation. Il s'agit en particulier des quartiers proches du lac Ponchartrain comme Lakeview le long du $17^{\text {th }}$ Street Canal, et surtout du Lower Ninth Ward, quartier « martyr » de l'ouragan et de la négligence des pouvoirs publics dans les représentations médiatiques américaines (fig. 1). Il ne s'agit pas ici de nier les destructions subies par ce quartier mais de montrer en quoi le tourisme macabre tel qu'il est pratiqué à la Nouvelle-Orléans participe d'une surreprésentation de cet espace. Le Lower Ninth Ward ne représente en effet que $3 \%$ de la surface de la ville, et avant l'ouragan, $2.9 \%$ de sa population. L'aspect particulièrement spectaculaire des destructions et une composition socio-raciale, avec une concentration de populations pauvres et noires, qui n'est pas la règle à l'échelle de la ville mais correspond aux discours sur la catastrophe propagés par les médias, en ont fait la vitrine des conséquences de Katrina à l'échelle des États-Unis. Ceci explique le surinvestissement d'acteurs nationaux sur ce seul quartier : les départements d'urbanisme de quatre universités américaines majeures (Harvard, Cornell, UCLA, Brown) y ont un projet d'étude en cours, artistes et troupes de théâtre mettent en scène les rues dévastées (En attendant Godot joué au pied de la levée par la Harlem Theater Compagny en octobre 2007) et plusieurs personnalités ultra-médiatiques (Bill Gates et surtout Brad Pitt) en ont fait le réceptacle de leurs dons économiques comme de leurs projets architecturaux et urbanistiques. Ces investissements multiples de la « scène » du Lower Ninth Ward sont validés par les touristes qui s'y rendent systématiquement et assurent également la diffusion des discours sur la catastrophe et la reconstruction dont cette scène est porteuse.

\section{VERS LA CONSTRUCTION D’UNE MÉMOIRE SÉLECTIVE DE LA CATASTROPHE}

Cette prégnance de certains quartiers dans la mémoire de la catastrophe enregistrée par les touristes peut être évaluée de deux manières. D’une part quand ces lieux sont aussi visités hors

7. D’après une série d'enquêtes menées par l'auteur à La Nouvelle-Orléans, en décembre 2006.

8. Une Second Line est la deuxième partie d'un Jazz funeral : alors que le cortège chemine d'abord lentement vers les cimetière au son d'un negro spiritual, le retour se fait sous forme d'une parade entrainante où tous sont conviés à célébrer la «joie de vivre » et le triomphe de la survie sur la mort. 
du cadre des tours, à l'initiative individuelle des touristes. Dans cette perspective, le Lower Ninth Ward subit une nouvelle fois les effets d'entraînement de sa médiatisation, puisqu'il est le seul quartier que la majorité des touristes interrogés durant l'hiver 2007 avaient " prévu » de visiter, y compris en s'y rendant par leurs propres moyens. D'autre part en posant la question de la « reconnaissance ${ }^{9}$ » de certains espaces auprès des visiteurs. Or sur les six noms de quartiers proposés, tous inondés à $90 \%$ ou plus en septembre 2005, un seul, le Lower Ninth Ward, bénéficiait d'une reconnaissance totale. Pour les autres moins d'une personne sur cinq (une sur dix pour Pontchartrain Park et Village de l'Est) avait déjà entendu parler de ces quartiers, avant ou durant leur visite à La Nouvelle-Orléans.

Cette sélectivité des espaces de mémoire de la catastrophe tels qu'ils sont pratiqués et diffusés par les touristes, et à travers eux par les représentations collectives américaines, pose bien sûr la question de l'oubli d'un certain nombre de lieux tout aussi concernés par les destructions consécutives à l'ouragan, mais aussi de problématiques liées aux processus de reconstruction. La majorité des quartiers inondés en septembre 2005 ne présentait pas, deux ans après, les dévastations photogéniques des lieux visités par les touristes. À Broadmoor comme Pontchartrain Park, Gentilly ou Eastern New Orleans, pas de maisons éventrées ni de voitures perchées dans les arbres, mais des habitations dont ne restent que les murs, le toit et la façade, s'alignant le long de rues désertes ou presque, sans éclairage public ni services d'aucune sorte. Les cloisons des maisons inondées ont en effet dû être abattues, (gutted), pour éviter le développement de moisissures toxiques. Ce travail de démolition a le plus souvent été effectués par des volontaires organisés en collectifs. Le travail de reconstruction des cloisons, de remise en état des réseaux, beaucoup plus coûteux, ne peut en revanche être réalisé que par des techniciens spécialisés, d'où le blocage des opérations au stade du démontage des cloisons. Le paysage offert aux passants est donc celui de constructions debout, alignées le long des trottoirs. Il faut s'approcher de très près ou s'y promener de nuit pour s'apercevoir que ces maisons sont complètements vides. Or ces lieux, où la composition socio-raciale de la population est souvent plus complexe que dans les quartiers «vitrines » de la catastrophe évoqués ci-dessus, ne bénéficient pas de la présence des touristes et du surcroît de visibilité que celle-ci peut apporter. Et s'ils sont le support de processus de mémorialisation spontanés de la catastrophe, les discours qu'ils produisent peinent à accéder à la sphère des débats publics, où leurs difficultés sont sous-représentées. Corrélativement, ce biais touristique dans la production d'une mémoire de la catastrophe et de ses conséquences à différents intervalles de temps conduit à la mise sous le boisseau de problématiques importantes relatives à la situation post-Katrina à La Nouvelle-Orléans. Le silence ménagé autour d’un taux de criminalité dramatique (le plus important à l'échelle nationale : 70 meurtres pour 100000 habitants en $2007^{10}$ ), qui affecte tous les quartiers de la ville, en est l'exemple le plus significatif. Jamais mentionné lors des visites touristiques, soigneusement ignoré par les brochures distribuées dans le French Quarter, l'accroissement de la violence urbaine, pourtant une conséquence directe des destructions occasionnées par l'ouragan, est passée sous silence pour ne pas perdre une clientèle qu'il faut certes informer mais surtout pas effrayer.

Pourtant, les circuits touristiques sont a priori un formidable vecteur d'informations sur l'état de la ville. Les pouvoirs publics eux-mêmes n’hésitent pas à présenter leurs rapports et revendications après avoir organisé un tour des quartiers détruits pour les représentants du gouvernement fédéral et des organismes en charge de la reconstruction. Le gouverneur de Louisiane, Kathleen Babineaux Blanco et le maire de la ville, C. Ray Nagin ont ainsi respectivement joué les « guides touristiques » pour les responsables de la FEMA et l'association des maires américains en septembre 2007. Cependant pour le grand public, la résilience attendue et affichée de la ville tend à limiter les discours catastrophistes ou revendicateurs sur l'évolution de la situation deux ans après

9. "Name recognition". Le questionnaire d'enquête demandait : « Have you ever heard the name of these neighborhoods before? ", pour les quartiers du Lower Ninth Ward, Lakeview, Gentilly, Broadmoor, Pontchartrain Park, Village de l'Est.

10. Source : New Orleans Police Department, 2007 Annual Report. 
la catastrophe. De sorte que les nécessités économiques qui sous-tendent le tourisme macabre amènent ce dernier à produire une mémoire sélective et consensuelle de la catastrophe.

\section{Mémorialisation ou muséification : enjeux et conflits d'acteurs autour de cette pratique}

Enfin, au-delà des conflits relatifs au contenu de la mémoire véhiculée par les acteurs de ce tourisme de catastrophe, c'est cette pratique elle-même qui est parfois violemment critiquée. Il est facile d'imaginer le malaise que peut créer le côté voyeuriste de ce tourisme, accepté par force de nécessité économique, comme le souligne le président du Bureau du Tourisme, Steven Perry : "Les visites des quartiers dévastés mettent tout le monde un peu mal à l'aise, [...] mais franchement, après tout ce qui s'est passé ici, cela semble absolument normal, et puis il faut bien vendre

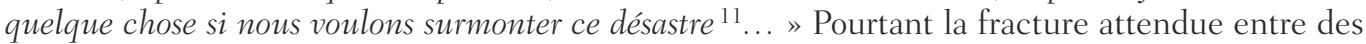
visiteurs à la curiosité "malsaine » et des locaux indignés que l'on transforme leurs souffrances en curiosité touristique n'apparaît pas si évidente. L'ambiguïté des motivations et des enjeux de cette pratique sont perçus des deux côtés. Ainsi Berner, un habitant de Gentilly dont la maison a sombré sous trois mètre d'eau souligne qu' « en tant que résident de La Nouvelle-Orléans, j'avais des sentiments conflictuels concernant ces "Katrina tours". J'avais l'impression que les guides profitaient de notre misère. Mais après avoir suivi l'un d'eux je les remercie de faire cela et d'éduquer les touristes, et moi aussi, sur ce qui s'est passé ici, et sur ce qui doit se passer pour que notre ville redevienne comme avant. " Ces propos recoupent singulièrement ceux de Wayne, un touriste venu du New Jersey: "J'étais un peu anxieux au début de faire du 'tourisme'dans ces quartiers détruits. Mais j'ai maintenant une connaissance de premier ordre sur ce qui s'est passé et j'ai l'intention de la partager avec d'autres pour obliger nos dirigeants à agir. » De fait les plus farouches opposants à ce tourisme macabre sont des acteurs extérieurs à la ville, responsables ou volontaires d’ONG s'étant donné pour mission de reconstruire les quartiers dévastés et d'assurer le retour de leurs habitants. Dans le Lower Ninth Ward, ce sont les membres du collectif Common Ground, fondé par un ex-Black Panther, Malik Rahim, qui s'en prennent parfois violemment aux visiteurs venus photographier le quartier dévasté. La confrontation passe par des accrochages verbaux mais surtout par des pancartes accrochées aux maisons en ruine rappelant que celles-ci sont des lieux de vie et non des monuments destinés aux touristes.

Car le fond de cette opposition au tourisme macabre ne concerne pas tant les discours que cette pratique permet de diffuser sur la catastrophe, mais sa tendance à la muséification des quartiers détruits, figés en lieux de mémoire. La pérennité de cette pratique suppose en effet le maintien du caractère spectaculaire de ces destructions et contredit donc les velléités de reconstruction des habitants ou des ONG impliqués dans ces lieux.

Le tourisme macabre à La Nouvelle-Orléans, en l'absence de mémorial officiel et durable, est donc soumis à une double temporalité. D’une part celle de l'attention portée par les touristes aux conséquences de la catastrophe. Or, deux ans après le passage de l'ouragan, l'intérêt des visiteurs s'émoussent, la couverture médiatique déclinante à l'échelle nationale, conduit à un

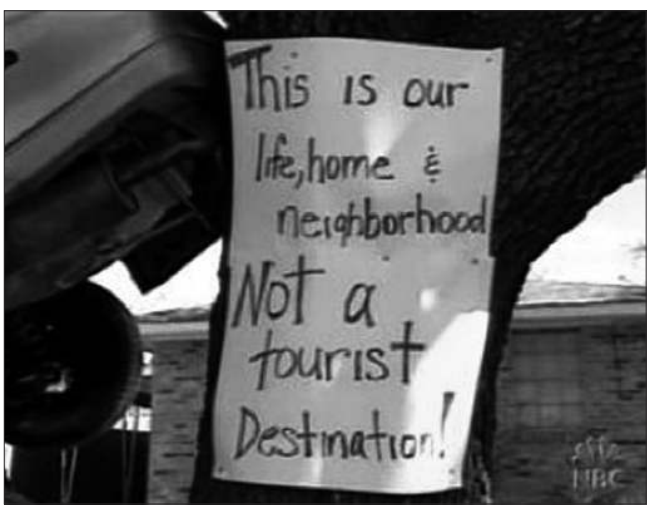

Photo 2 : «C'est notre vie, notre maison et notre quartier, pas une attraction pour touristes! » (Lower Ninth Ward, mars 2007 - cliché de l'auteur)

"This is our life, our home and our neighborhood, not a tourist destination" 
déclin de la demande de visite des quartiers encore détruits. Les visites guidées classiques de la ville ont repris le pas sur les Katrina tours dans les activités des acteurs de l'industrie touristique. D'autre part, le progressif effacement des dévastations dans l'espace urbain contribue à la disparition des lieux vitrines de Katrina et de sa mémoire. Les maisons les plus spectaculairement détruites du Lower Ninth Ward, à proximité de la levée de l'Industrial Canal, ont été rasées durant l'été 2006, et l'ancien cimetière urbain est redevenu un marais de hautes herbes. Dans le quartier de Lakeview, les reconstructions menées à l'initiative des habitants ont rapidement progressée à l'ombre de la levée à peine réparée, et là aussi, les traces les plus spectaculaires de Katrina ont été effacées de la mémoire des paysages urbains offerts aux touristes.

\section{Conclusion}

Ces évolutions interrogent la nature profonde de ce tourisme macabre comme rapport spectaculaire ou comme rapport identitaire à la ville. La première dimension renvoie à une perspective postmoderniste mettant l'accent sur l'importance des signes visuels et des discours sur la catastrophe, mais aussi sur leur caractère éphémère, cette pratique relevant donc d'un tourisme « évènementiel » soumis aux fluctuations de la mémoire collective. Dans ce contexte postmoderne des usages de l'espace nous assisterions à une commodification de la tragédie à des fins de profit économique et de divertissement.

Cette analyse et d'autres études de cas semblables tendent à dénoncer la détérioration des sites de catastrophes inhérente à ce type de mise en tourisme (Weeks, 2003 ; Yuill, 2004). Détérioration ici ne signifie pas nécessairement dégradation physique des sites. Il s'agit aussi de la transformation d'un lieu "ancré », aux fonctionnements et aux significations complexes, en un espace «traversé » et monofonctionnel, support et non plus vécu, ce qui entraîne de sérieuses dissonances entre les intérêts des touristes et ceux des locaux, "producteurs » habituels de cet espace. La question du développement d'un rapport identitaire, c'est-à-dire d'un dépassement de la passivité consommatrice vers une implication dans la production de l'espace urbain et de ses significations, semble passer par la réduction de cette dissonance et par la construction partagée d'une mémoire de la catastrophe.

Ce tourisme macabre participe finalement de logiques géographiques connues, celles de la patrimonialisation de l'espace, mais il les complexifie en introduisant de nouveaux rapports entre les acteurs, en interrogeant la mise en scène des sites de tragédie humaine, et en reposant la question des temporalités de la mémoire des lieux, intégrée et digérée dans les dynamiques de production des espaces urbains.

\section{Bibliographie}

BNOB, 2006. - Report of the cultural committee of the Mayor's Bring New Orleans Back Commission. 14 p. [http://www.aeaconsulting.com/site/bnob/BNOB \%20EXECUTIVE \%20SUMMARY \%2020060202.pdf]

Eyre (A.), Wachtendorf (T.), WebB (G.), 2000. - « The popular culture of disaster », International Journal of Mass Emergencies and Disasters, special issue, vol. 18, $\mathrm{n}^{\circ}$ 1, p. 1-115

Foley (M.), Lennon (J.), 2000. - Dark Tourism: the Attraction of Death and Disaster, Londres/New York, Continuum, $256 \mathrm{p}$.

Halbwachs (M.), 1992.- On Collective Memory, Chicago, The University of Chicago Press, 254 p.

HaYden (D.), 1995. - The Power of Space : Urban Landscapes as Public History, Cambridge, The MIT Press, $356 \mathrm{p}$.

Hernandez (J.), 2006. - Katrina : une tragédie américaine?, Actes du Festival International de Géographie de Saint-Dié [http://fig-st-die.education.fr/actes/actes_2006/hernandez/article.htm]. 
Lewis (P.), 2003. - New Orleans. The Making of an Urban Landscape, Charlottesville (VA), The University of Virginia Press, 200 p.

Linenthal (E.), 2005. - «The predicament of aftermath: Oklahoma City and September 11 th. », dans Campanella (T.), Vale (L. J.) (dir.), The Resilient City: How Modern Cities Recover from Disasters, New York, Oxford University Press, p. 55-74.

LipzTig (G.), 2001. - Time Passages : Collective Memory and American Popular Culture, Minneapolis, The University of Minnesota Press, 328 p.

Mitchell (D.), 2000. - Cultural Geography. A Critical Introduction, Oxford, Blackwell. 325 p.

SEATON (T.), 1996. - « From Thanatopsis to Thanatourism : Guided by the Dark », Journal of International Heritage Studies, n 22, p. 234-244.

STANONIS (A.), 2006. - Creating the Big Easy : New Orleans and the emergence of modern tourism. 1918-1945, Athens, University of Georgia Press, 317 p.

Stein (H.), 2003. - « Days of Awe : September 11 th and its cultural psychodynamics », Journal for the Psychoanalysis of Culture and Society, vol. 8, n² 2, p. 187-199.

Stone (P. R.), 2006. - «A Dark Tourism Spectrum : towards a typology of macabre related tourist sites, attractions, and exhibitions », Tourism : An Interdisciplinary International Journal, vol. 54, n 2, p. $145-$ 160.

Weeks (J.), 2003. - Gettysburg. Memory, Market and American Shrine, Princeton (NJ), Princeton University Press, $288 \mathrm{p}$.

YuiLL (S.), 2004. - Dark Tourism : understanding visistor motivation at sites of death and disaster, Dallas, Texas A\&M University, 263 p.

Cet article a été reçu le 12 mars 2008 et définitivement accepté le 17 septembre 2008. 
